\title{
Diagnósticos de enfermagem em casos de COVID-19 com evolução clínica para sepse
}

\author{
Nursing diagnoses in COVID-19 cases with clinical evolution for sepsis \\ Diagnóstico de enfermería en casos de COVID-19 con evolución clínica de sepsis
}

Recebido: 15/12/2020 | Revisado: 24/12/2020 | Aceito: 05/01/2021 | Publicado: 06/01/2021

\author{
Maria Isabel Caetano da Silva \\ ORCID: https://orcid.org/0000-0001-5389-3664 \\ Universidade Regional do Cariri, Brasil \\ E-mail: mariaisabelcs28@outlook.com \\ Raul Roriston Gomes da Silva \\ ORCID: https://orcid.org/0000-0002-8576-5875 \\ Universidade Regional do Cariri, Brasil \\ E-mail: roriston@live.com \\ Rayane Moreira de Alencar \\ ORCID: https://orcid.org/0000-0003-1989-3200 \\ Universidade Regional do Cariri, Brasil \\ E-mail: rayanealencar@hotmail.com \\ Naftale Alves dos Santos Gadelha \\ ORCID: https://orcid.org/0000-0001-6257-9431 \\ Universidade Regional do Cariri, Brasil \\ E-mail: naftalealves@yahoo.com.br \\ Woneska Rodrigues Pinheiro \\ ORCID: https://orcid.org/0000-0003-3353-9240 \\ Universidade Regional do Cariri, Brasil \\ E-mail:woneskar@gmail.com
}

\begin{abstract}
Resumo
Objetivo: elaborar Diagnósticos de Enfermagem (DE), através da identificação de sinais e sintomas descritos na literatura de pacientes com COVID-19 com evolução clínica para sepse ou choque séptico. Método: Trata-se de uma revisão narrativa da literatura onde a busca ocorreu nas bases de dados: Medical Literature Analysis and Retrieval System Online (MEDLINE) (via PubMed), Cumulative Index to Nursing and Allied Health Literature (CINAHL), Cochrane e EMBASE, nos meses de abril e maio de 2020. Utilizou-se a estratégia de busca avançada para realização do cruzamento entre os MeSH a partir do operador booleano AND: Coronavirus Infections AND Sepsis, obtendo-se amostra final de 8 relatos de casos. Resultados: Mediante os estudos, foram elencados 16 diagnósticos de enfermagem da NANDA-I de acordo com o quadro clínico apresentado pelos pacientes, destes, seis mostraram-se mais prevalentes, sendo intitulados de ventilação espontânea prejudicada, padrão respiratório ineficaz, risco de desequilíbrio eletrolítico, termorregulação ineficaz, volume de líquidos deficiente e dor aguda. Conclusão: A identificação desses DE oferece subsídios para elaboração de um cuidar direcionado a atender as necessidades dos pacientes, ademais, valoriza a profissão como ciência e guia os profissionais de enfermagem para um cuidar organizado e com qualidade.
\end{abstract}

Palavras-chave: Infecção por coronavírus; Sepse; Choque séptico; Diagnóstico de enfermagem; COVID-19.

\begin{abstract}
Objective: to elaborate Nursing Diagnoses (DE), through the identification of signs and symptoms described in the literature of patients with COVID-19 with clinical evolution for sepsis or septic shock. Method: This is a narrative review of the literature where the search took place in the databases: Medical Literature Analysis and Retrieval System Online (MEDLINE) (via PubMed), Cumulative Index to Nursing and Allied Health Literature (CINAHL), Cochrane and EMBASE, in the months of April and May 2020. The advanced search strategy was used to perform the crossing between MeSH using the Boolean operator AND: Coronavirus Infections AND Sepsis, obtaining a final sample of 8 case reports. Results: Through studies, 16 NANDA-I nursing diagnoses were listed according to the clinical picture presented by the patients, of these, six were more prevalent, being entitled to impaired spontaneous ventilation, ineffective breathing pattern, risk of electrolyte imbalance, ineffective thermoregulation, deficient fluid volume and acute pain. Conclusion: The identification of these ND offers subsidies for the elaboration of care directed to meet the needs of patients, in addition, it values the profession as a science and guides nursing professionals towards organized and quality care.
\end{abstract}

Keywords: Infection from coronavirus; Sepsis; Septic shock; Nursing Diagnosis; COVID-19.

\section{Resumen}

Objetivo: elaborar Diagnósticos de Enfermería (DE), mediante la identificación de signos y síntomas descritos en la literatura de pacientes con COVID-19 con evolución clínica para sepsis o shock séptico. Método: Esta es una revisión 
narrativa de la literatura donde se realizó la búsqueda en las bases de datos: Sistema de recuperación y análisis de literatura médica en línea (MEDLINE) (a través de PubMed), Índice acumulativo de literatura de enfermería y salud aliada (CINAHL), Cochrane y EMBASE, en los meses de abril y mayo de 2020. La estrategia de búsqueda avanzada se utilizó para realizar el cruce entre MeSH utilizando el operador booleano AND: Infecciones por coronavirus Y sepsis, obteniendo una muestra final de 8 informes de casos. Resultados: a través de los estudios, se enumeraron 16 diagnósticos de enfermería NANDA-I de acuerdo con el cuadro clínico presentado por los pacientes, de estos, seis fueron más prevalentes, teniendo derecho a ventilación espontánea deteriorada, patrón de respiración ineficaz, riesgo de desequilibrio electrolítico , termorregulación ineficaz, volumen de líquido deficiente y dolor agudo. Conclusión: La identificación de estos ND ofrece subsidios para la elaboración de atención dirigida a satisfacer las necesidades de los pacientes, además, valora la profesión como ciencia y guía a los profesionales de enfermería hacia una atención organizada y de calidad.

Palabras clave: Infección por coronavirus; Septicemia; Shock séptico; Diagnóstico de enfermeira; COVID-19.

\section{Introdução}

Em dezembro de 2019, em Wuhan na China, foram identificados os primeiros casos de doenças causadas pelo novo coronavírus, a COVID-19 como também é denominada. Trata-se de uma doença causada pelo vírus SARS-CoV-2, responsável por provocar principalmente, comprometimento respiratório. O surto atingiu grandes dimensões e, em janeiro de 2020 foi declarado pela Organização Mundial de Saúde (OMS) estado de pandemia (Lee, Ng \& Khong, 2020; Organização PanAmericana de Saúde \& Organização Mundial da Saúde, 2020).

Devido à alta transmissibilidade da doença, no mundo, a quantidade de pessoas contaminadas ultrapassa 5.175.925 casos, com mais de 338.089 mortes, os casos confirmados estão distribuídos em 185 países e todos os continentes já foram atingidos. Até 23 de maio de 2020, os Estados Unidos apresentava o maior número de casos, com 1.601 .434 pessoas infectadas. O Brasil, está em $2^{\circ}$ lugar no ranking dos países com maior número de casos confirmados da doença e em $6^{\circ}$ no número de mortes, excedendo 347.398 casos e 22.013 óbitos (Associação Mineira de Epidemiologia e Controle de Infecções, 2020; Ministério da Saúde, 2020).

Acredita-se que a transmissão do vírus entre as pessoas ocorre principalmente por meio de gotículas respiratórias oriundas de indivíduos infectados, esses, ao espirrar ou tossir, libera no ambiente partículas respiratórias com a presença de vírus que se espalha pelo ar e contamina outras pessoas. Assim, a disseminação acontece pelo contato próximo entre pessoas, semelhante ao contágio por outros vírus, como a influenza. Ademais, superfícies e objetos contaminados também funcionam como veículos de propagação do vírus (Ministério da Saúde, 2020).

A doença possui alta capacidade de transmissão. A manifestação e evolução clínica no paciente difere entre os casos, apresentando desde a forma assintomática; casos de sintomas leves com quadro clínico de resfriado e febre; à mais graves com manifestações de desconforto respiratório, frequência respiratória elevada e saturação de oxigênio < $93 \%$ em ar ambiente, nesses últimos, os pacientes necessitam de cuidados intensivos. Essas mudanças na apresentação da doença acontecem devido a presença de alguns fatores que contribuem para o agravamento dos danos provocados pelo vírus, especificamente: idade, comprometimento imunológico e doenças crônicas prévias (Dias et al., 2020).

Neste contexto, a sepse foi detectada em uma parcela considerável dos pacientes com COVID-19 que foram a óbito e em parte dos sobreviventes que estavam internados na Unidade de Terapia Intensiva (UTI). Esses achados se originaram da avaliação de indicadores em exames coletados dos pacientes que quando era identificado alterações sugeriam a falha de algum órgão. A título de exemplo, a contagem reduzida de plaquetas; alterações no marcador da função renal, a creatinina; e elevação do valor de dímero-D no sangue, fragmentos de proteínas que estão relacionadas ao processo de coagulação (Alvim, 2020).

A sepse é definida como qualquer disfunção orgânica originada de uma resposta exacerbada do indivíduo a infecção, uma síndrome clínica descrita pela existência de mecanismos inflamatórios, onde desencadeiam alterações celulares e circulatórias, apresentando vasodilatação, aumento da permeabilidade capilar, hipovolemia, hipotensão e coagulação intravascular disseminada (CID) (Dewitte et al., 2017; Zonta et al., 2018). 
Assim como para assistência a pacientes com sepse, os cuidados a saúde de pacientes vítimas da COVID-19 exigem equipe especializada. Dentre os profissionais que compõem a equipe que atuam na linha de frente no combate a atual pandemia encontra-se o enfermeiro que é o profissional que permanece maior parte do tempo ao lado do paciente e precisa estar preparado para prestar assistência contínua com qualidade. Neste contexto, o Processo de Enfermagem (PE) é a base para a sustentação da Sistematização da Assistência de Enfermagem, sua utilização nos serviços de saúde é fundamental na identificação dos sinais e sintomas dos problemas de saúde provocados pela COVID-19 (Conselho Federal de Enfermagem, 2020; Pimentel, 2019).

O Diagnóstico de Enfermagem (DE) segunda fase do PE, consiste em agrupar informações coletadas a partir da realização da anamnese e exame físico do paciente referente a resposta humana que requer intervenção de enfermagem. É ainda definido como julgamento clínico das respostas do indivíduo, família ou comunidade relacionado a situações vitais no qual o enfermeiro é responsável por assistir (Ferreira et al., 2016; Martins, 2014).

Portanto, é importante o profissional enfermeiro conhecer os diagnósticos de enfermagem apresentados na taxonomia utilizada no Brasil para os pacientes que estão internados com COVID-19 e apresentam quadro de sepse, promovendo padronização no cuidado a esses pacientes. Com base no exposto, o objetivo do estudo consiste em elaborar diagnósticos de enfermagem, segundo a NANDA-I (2018- 2020), através da identificação de sinais e sintomas descritos na literatura de pacientes com COVID-19 com evolução clínica para sepse ou choque séptico.

\section{Metodologia}

Trata-se de uma revisão narrativa da literatura, de cunho exploratório com abordagem qualitativa.

A revisão narrativa consiste em uma análise ampla de estudos com o objetivo de descrever e contextualizar sobre um assunto específico (Botelho, Cunha \& Macedo, 2011). Estudos exploratórios com abordagem qualitativa possibilita a compreensão do fenômeno em estudo a partir de uma análise mais aprofundada das informações (Pereira et al., 2018).

A formulação da pergunta foi inspirada na estrutura PICO (acrônimo para patient, intervention, comparison, outcomes). A utilização dessa estratégia permite identificar termos que auxiliem na localização de estudos relevantes para compor a bases de dados da revisão (Fineout-Overholt \& Stillwell, 2011).

A questão de investigação que norteou este estudo foi: "Quais os sinais e sintomas que podem subsidiar a inferência de diagnósticos de enfermagem em casos de COVID-19 com evolução clínica para sepse ou choque séptico?”. Nela, o elemento (P) consiste nos pacientes com COVID-19 com evolução clínica para sepse ou choque séptico; (I) sinais e sintomas; e o elemento $(\mathrm{O})$ diagnósticos de enfermagem. Ressalta-se que, dependendo do método de revisão, não se emprega todos os elementos da estratégia PICO. Nesta revisão narrativa, o terceiro elemento (C), ou seja, a comparação, não foi utilizada.

A coleta de dados ocorreu em abril e maio de 2020 nas bases de dados Medical Literature Analysis and Retrieval System Online (MEDLINE) (via PubMed), Cumulative Index to Nursing and Allied Health Literature (CINAHL), Cochrane e EMBASE; a procura nas bases se deu através do método de busca avançada e utilizando como estratégia de investigação o cruzamento entre os Descritores em Ciências da Saúde (DeCS) e seus respectivos MeSH a partir do operador booleano AND: Coronavirus Infections AND Sepsis; que resultou em 441 publicações.

Os filtros aplicados foram: Texto completo disponível; gratuito; idiomas português, inglês e espanhol; tipo de documento artigo e não houve restrições quanto ao ano de publicação.

Foram incluídos somente relatos de casos que abordam as caraterísticas clínicas de pacientes com COVID-19 que evoluíram para sepse e que relatam as necessidades humanas básicas afetadas em pacientes com o novo coronavírus e sepse ou choque séptico relatadas no momento da admissão e durante a avaliação clínica dos pacientes pela equipe de saúde. Foram 
excluídos desta pesquisa artigos duplicados, capítulos de livros, diretrizes, manuais, estudos de monografias, teses e dissertações.

Após aplicação dos filtros, a seleção dos artigos ocorreu em duas etapas: A primeira com a leitura de títulos e resumos dos 183 artigos que restaram do processo de filtragem, observando a relação do artigo com a temática pesquisada e sua adequação aos critérios de inclusão. Desta forma, foram selecionados 17 artigos; estes foram recuperados e lidos na íntegra para delimitação do corpus de análise, chegando-se a um total de 8 estudos para compor a amostra.

A busca foi realizada em pares e o desempate para decidir a inclusão ou a exclusão de determinados estudos ficou como responsabilidade de um terceiro pesquisador.

O processo de seleção e triagem dos artigos está representado em fluxograma, elaborado com base no Preferred Reporting Items for Systematic Reviews and Meta-Analyses (PRISMA) (Moher et al., 2015), que consiste em uma lista de verificações e facilita a demonstração das etapas da revisão (Figura 1).

Figura 1 - Fluxograma da seleção dos estudos segundo o Preferred Reporting Items for Systematic Reviews and MetaAnalyses (PRISMA 2015). Crato, Ceará, Brasil, 2020.
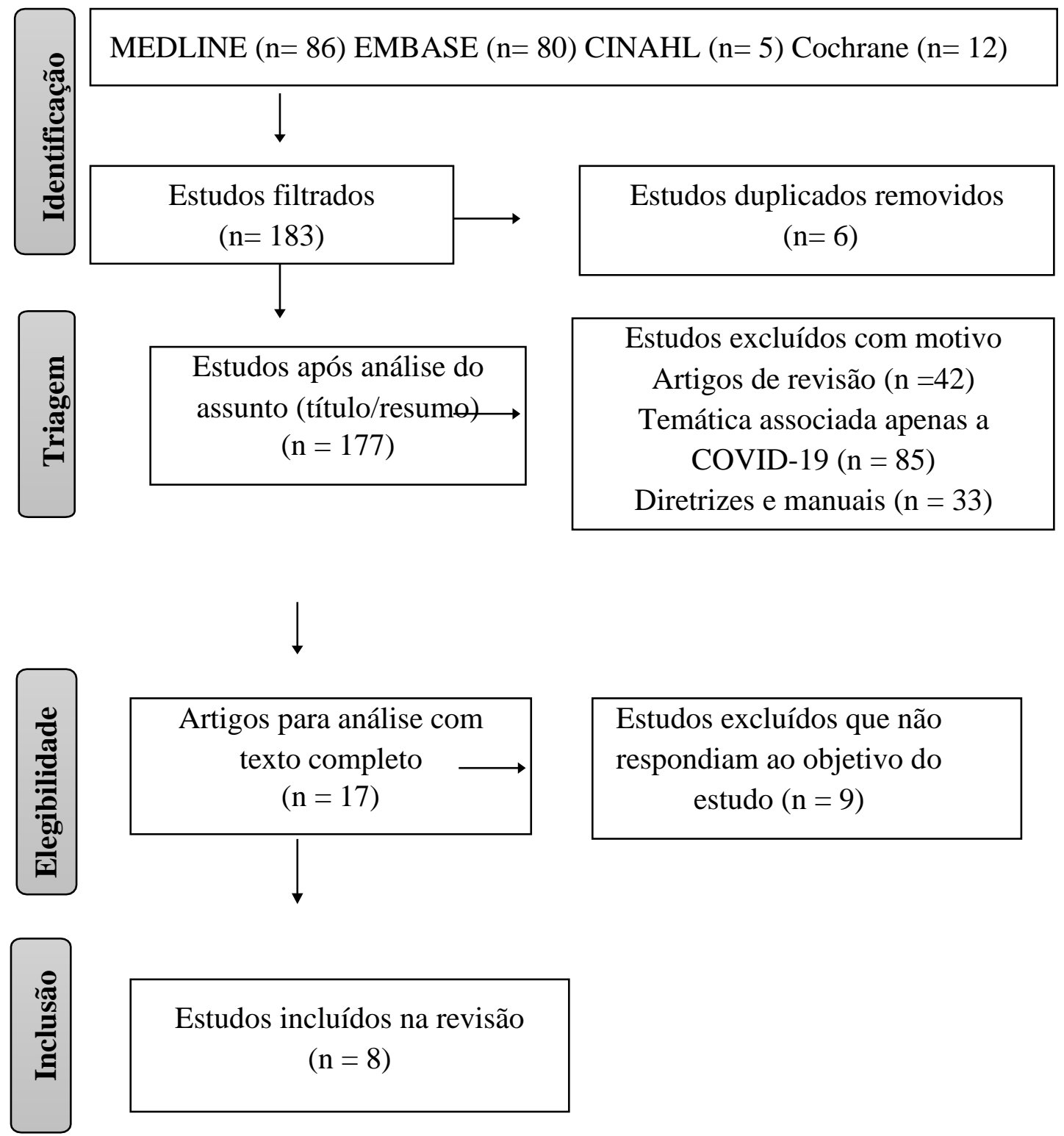

Fonte: Autores. 
Os dados foram analisados em pares utilizando a literatura pertinente, organizados e apresentados em quadros contemplando as informações: Autores/ano de publicação, título, objetivo, população do estudo, local onde o estudo foi realizado e principais achados; bem como sinais e sintomas apresentados por pacientes com COVID-19 e sepse ou choque séptico.

Em posse dos sinais e sintomas encontrados nos estudos, os DEs foram elaborados tomando por referência a taxonomia da NANDA I versão mais atual 2018-2020. Todas as manifestações clínicas foram listadas buscando semelhanças com as definições dos diagnósticos de enfermagem, características definidoras e fatores relacionados.

Ademais, os achados foram descritos e discutidos de forma crítica e reflexiva, conciliando com as publicações relacionadas ao tema de estudo.

\section{Resultados}

Após a leitura completa dos artigos, foram selecionados 8 relatos de casos, os quais passaram por análise e coleta das informações de identificação dos artigos: Autores, ano de publicação, título, objetivo (Quadro 1); população do estudo, local, principais achados (Quadro 2); e as características clínicas comumente apresentadas por pacientes com a COVID-19 que evoluíram para sepse (Quadro 3). Ressalta-se que todos possuem idioma inglês e estão indexados na base de dados MEDLINE.

Quadro 1 - Características dos artigos selecionados de acordo com autores/ano de publicação, título e objetivo. Crato, Ceará, Brasil, 2020.

\begin{tabular}{|c|c|c|}
\hline $\begin{array}{l}\text { Autores/ ano de } \\
\text { publicação }\end{array}$ & Título & Objetivo \\
\hline $\begin{array}{l}\text { Aghdam, Jafari, \& } \\
\text { Eftekhari (2020) }\end{array}$ & $\begin{array}{l}\text { Novel coronavirus in a } 15 \text {-day-old neonate } \\
\text { with clinical signs of sepsis, a case report }\end{array}$ & $\begin{array}{l}\text { Apresentar o caso de um neonato de } 15 \\
\text { dias diagnosticado com covid-19 com } \\
\text { sinais clínicos de sepse. }\end{array}$ \\
\hline Chen et al. (2020) & $\begin{array}{l}\text { Clinical characteristics of } 113 \text { deceased } \\
\text { patients with coronavirus } \\
\text { disease 2019: retrospective study }\end{array}$ & $\begin{array}{l}\text { Delinear as características clínicas de } \\
\text { pacientes com covid-19 que foram a } \\
\text { óbito. }\end{array}$ \\
\hline Ferreira et al. (2020) & $\begin{array}{l}\text { Critically ill SARS-CoV-2-infected patients } \\
\text { are not stratified as sepsis by the qSOFA }\end{array}$ & $\begin{array}{l}\text { Examinar se o ponto 2, limiar de qSOFA } \\
\text { é um escore clínico adequado para } \\
\text { classificar sepse em pacientes com } \\
\text { Covid-19. }\end{array}$ \\
\hline Hansen et al. (2020) & $\begin{array}{l}\text { COVID-19: Symptoms, course of illness } \\
\text { and use of clinical scoring systems for } \\
\text { the first } 42 \text { patients admitted to a } \\
\text { Norwegian local hospital }\end{array}$ & $\begin{array}{l}\text { Apresentar as características, sintomas da } \\
\text { COVID-19, evolução da doença e uso de } \\
\text { sistemas de pontuação clínica para os } \\
\text { primeiros pacientes internados em um } \\
\text { hospital local Norueguês. }\end{array}$ \\
\hline Hong et al. (2020) & $\begin{array}{l}\text { Clinical Features and Outcomes of } 98 \\
\text { Patients Hospitalized with SARS-CoV-2 } \\
\text { Infection in Daegu, South Korea: A Brief } \\
\text { Descriptive Study }\end{array}$ & $\begin{array}{l}\text { Descrever as características clínicas e } \\
\text { resultados de pacientes hospitalizados } \\
\text { com SARS-CoV-2. }\end{array}$ \\
\hline Munoz et al. (2020) & $\begin{array}{l}\text { Late-Onset Neonatal Sepsis in a Patient with } \\
\text { Covid-19 }\end{array}$ & $\begin{array}{l}\text { Relatar o caso de sepse neonatal de um } \\
\text { paciente com COVID- } 19 .\end{array}$ \\
\hline Zhang (2020) & $\begin{array}{l}\text { Imaging changes in severe COVID-19 } \\
\text { pneumonia in advanced stage }\end{array}$ & $\begin{array}{l}\text { Relatar o caso de um homem } \\
\text { diagnosticado com pneumonia grave por }\end{array}$ \\
\hline
\end{tabular}




\begin{tabular}{|l|l|l|}
\hline & & $\begin{array}{l}\text { COVID-19 combinada com síndrome do } \\
\text { desconforto respiratório agudo (SDRA), } \\
\text { choque séptico e síndrome da disfunção } \\
\text { de múltiplos órgãos. }\end{array}$ \\
\hline Zhou et al. (2020) & $\begin{array}{l}\text { Clinical course and risk factors for mortality } \\
\text { of adult } \\
\text { inpatients with COVID-19 in Wuhan, China: } \\
\text { a retrospective } \\
\text { cohort study }\end{array}$ & $\begin{array}{l}\text { Relatar os fatores de risco para } \\
\text { mortalidade por COVID-19, curso } \\
\text { clínico da doença e disseminação viral. }\end{array}$ \\
\hline
\end{tabular}

Fonte: Autores.

Quadro 2 - Características dos artigos selecionados de acordo com população do estudo, local e principais achados. Crato, Ceará, Brasil, 2020.

\begin{tabular}{|c|c|c|c|}
\hline $\begin{array}{c}\text { Autores/ano de } \\
\text { publicação }\end{array}$ & $\begin{array}{l}\text { População do } \\
\text { estudo }\end{array}$ & Local & Principais achados \\
\hline $\begin{array}{l}\text { Aghdam, Jafari, } \\
\& \quad \text { Eftekhari } \\
(2020)\end{array}$ & $\begin{array}{l}\text { Um neonato de } 15 \\
\text { dias de idade }\end{array}$ & Irã & $\begin{array}{l}\text { Neonato na admissão hospitalar apresentava febre } \\
\left(38,2^{\circ} \mathrm{c}\right) \text {, taquicardia ( } 170 \text { batimentos/min.), taquipneia } \\
(66 \mathrm{irpm}) \text {, retração subcostal e saturação de oxigênio de } \\
93 \% \text {. Paciente foi transferido para UTI devido a } \\
\text { necessidade de suporte respiratório e gravidade do } \\
\text { quadro, foi tratado com fluidoterapia adequada, } \\
\text { oxigenoterapia, antibiótico e antivirais. }\end{array}$ \\
\hline 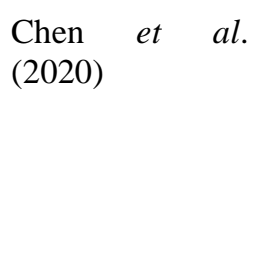 & $\begin{array}{l}113 \text { pacientes com } \\
\text { COVID-19 que } \\
\text { evoluíram para } \\
\text { óbito }\end{array}$ & $\begin{array}{l}\text { Wuhan } \\
\text { (China) }\end{array}$ & $\begin{array}{l}\text { As complicações encontradas nos pacientes com } \\
\text { COVID-19 que evoluíram para óbito foram: SDRA } \\
(100 \%) \text {, insuficiência respiratória }(51 \%) \text {, sepse }(100 \%) \text {, } \\
\text { lesão cardíaca aguda }(77 \%) \text {, insuficiência cardíaca } \\
(49 \%) \text {, lesão renal aguda }(25 \%) \text { e encefalopatia } \\
\text { hipóxica }(20 \%) \text {. }\end{array}$ \\
\hline $\begin{array}{l}\text { Ferreira et al. } \\
(2020)\end{array}$ & $\begin{array}{l}52 \text { pacientes } \\
\text { críticos internados } \\
\text { na UTI } \\
\text { com infecção } \\
\text { confirmada por } \\
\text { SARS-CoV-2 }\end{array}$ & França & $\begin{array}{l}38(73 \%) \text { pacientes foram submetidos a ventilação } \\
\text { mecânica }(\mathrm{VM}) \text {, desses, } 27(87 \%) \text { pacientes tinham } \\
\text { qSOFA } \leq 1 \text { e } 4 \text { pacientes tiveram qSOFA de } 2 \text { pontos; } \\
14(27 \%) \text { pacientes receberam alta sem necessitar de } \\
\text { suporte ventilatório e todos tiveram qSOFA } \leq 1 \text {. Todos } \\
\text { os pacientes com qSOFA }=1 \text { assim foram pontuados } \\
\text { por apresentarem frequência respiratória }(\mathrm{FR}) \geq 22 / \\
\text { min. e os que tiveram qSOFA }=2 \text { além de pontuarem a } \\
\text { FR também apresentaram pressão arterial sistólica } \leq \\
100 \mathrm{mmHg} \text {. }\end{array}$ \\
\hline $\begin{array}{l}\text { Hansen } \\
(2020)\end{array}$ & $\begin{array}{l}42 \text { pacientes } \\
\text { internados com } \\
\text { COVID-19 em um } \\
\text { hospital } \\
\text { Norueguês }\end{array}$ & Noruega & $\begin{array}{l}65 \% \text { dos pacientes eram do sexo masculino, maioria } \\
(67 \%) \text { tinham idade } \geq 70 \text { anos e possuíam boa } \\
\text { capacidade funcional, diabetes mellitus foi a condição } \\
\text { comórbida mais comum }(16,2 \%) \text {. } 21 \% \text { dos pacientes } \\
\text { apresentaram a forma grave da doença, } 11 \% \text { tinham FR } \\
\geq 22 / \text { min, } 12 \% \text { pontuaram um no escore qSOFA e } 3 \\
\text { pacientes apresentaram pontuação } \geq 2,11 \% \text { dos } \\
\text { pacientes atenderam a mais de dois critérios de SIRS. }\end{array}$ \\
\hline $\begin{array}{l}\text { Hong } \\
(2020)\end{array} \quad$ et $\quad$ al. & $\begin{array}{l}98 \text { pacientes } \\
\text { hospitalizados } \\
\text { com infecção por } \\
\text { SARS-CoV-2 na } \\
\text { Coréia do Sul }\end{array}$ & $\begin{array}{l}\text { Coréia do } \\
\text { Sul }\end{array}$ & $\begin{array}{l}\text { Maioria dos pacientes }(61,2 \%) \text { eram do sexo feminino e } \\
(40,8 \%) \text { tinham idade de } 50-64 \text { anos, hipertensão foi a } \\
\text { comorbidade mais prevalente }(30,6 \%) \text { e os sinais e } \\
\text { sintomas apresentados no momento da admissão foram } \\
\text { principalmente: Febre, tosse, escarro, mialgia e } \\
\text { dispneia. As complicações observadas durante o curso } \\
\text { da doença consistiram em SDRA }(18,3 \%) \text {, choque }\end{array}$ \\
\hline
\end{tabular}




\begin{tabular}{|c|c|c|c|}
\hline & & & $\begin{array}{l}\text { séptico }(9,2 \%) \text {, lesão cardíaca aguda }(11,2 \%) \text { e lesão } \\
\text { renal aguda }(9,2 \%) \text {. Todos os pacientes com choque } \\
\text { séptico necessitaram de tratamento na UTI. }\end{array}$ \\
\hline $\begin{array}{l}\text { Munoz et al. } \\
(2020)\end{array}$ & $\begin{array}{l}\text { Um neonato de } \\
\text { três semanas com } \\
\text { quadro de sepse e } \\
\text { infecção } \\
\text { confirmada para } \\
\text { COVID-19 }\end{array}$ & $\begin{array}{l}\text { Houston } \\
\text { (Estados } \\
\text { Unidos) }\end{array}$ & $\begin{array}{l}\text { As características clínicas do neonato no momento da } \\
\text { admissão eram: Temperatura de } 36,1^{\circ} \mathrm{c} \text {, frequência } \\
\text { cardíaca } 166 \text { batimentos/min., pressão arterial } 89 / 63 \\
\mathrm{mmHg} \text {, FR }=40 \text { respirações/min. e saturação de } \\
\text { oxigênio } 87 \% \text { em ar ambiente. O paciente precisou ser } \\
\text { transferido para uma unidade de terapia intensiva } \\
\text { pediátrica, durante o processo de transferência, } \\
\text { apresentou hipotensão, taquicardia, hipotermia e } \\
\text { taquipneia. }\end{array}$ \\
\hline Zhang (2020) & $\begin{array}{l}\text { Homem de } 75 \\
\text { anos, confirmado } \\
\text { com pneumonia } \\
\text { grave por COVID- } \\
19 \text { em tratamento } \\
\text { na unidade de } \\
\text { terapia intensiva } \\
\text { (UTI) }\end{array}$ & China & $\begin{array}{l}\text { Paciente revelou temperatura corporal de } 35,5^{\circ} \mathrm{c} \text {, } \\
\text { frequência cardíaca de } 54 \text { batimentos } / \mathrm{min} \text {., pressão } \\
\text { arterial } 136 / 79 \mathrm{mmHg} \text { (noradrenalina } 0,2 \mu \mathrm{g} / \mathrm{kg} / \mathrm{min} \text { ), } \\
\text { saturação de oxigênio } 91 \% \text {, foi diagnosticado com } \\
\text { pneumonia grave por COVID- } 19 \text { combinado com } \\
\text { SDRA, choque séptico e síndrome da disfunção de } \\
\text { múltiplos órgãos }\end{array}$ \\
\hline $\begin{array}{l}\text { Zhou et al. } \\
(2020)\end{array}$ & $\begin{array}{l}191 \quad \text { pacientes } \\
\text { internados com } \\
\text { COVID-19 }\end{array}$ & $\begin{array}{l}\text { Wuhan } \\
\text { (China) }\end{array}$ & $\begin{array}{l}\text { A idade média dos pacientes era de } 56 \text { anos, maioria era } \\
\text { do sexo masculino }(62 \%) \text {, a comorbidade mais presente } \\
\text { foi a hipertensão }(30 \%) \text {, seguida de diabetes mellitus } \\
(19 \%) \text { e doenças coronariana }(8 \%) \text {. Do desfecho } \\
\text { clínico, } 59 \% \text { dos pacientes evoluíram com quadro de } \\
\text { sepse, } 54 \% \text { com parada cardiorrespiratória, } 31 \% \text { com } \\
\text { SDRA, } 23 \% \text { com insuficiência cardíaca e } 20 \% \text { com } \\
\text { choque séptico. }\end{array}$ \\
\hline
\end{tabular}

Fonte: Autores.

As manifestações clínicas prevalentes nos pacientes dos estudos selecionados foram febre, tosse, dispneia, diminuição da saturação de oxigênio, hipotensão, vômito e diarreia (Chen et al., 2020; Ferreira et al., 2020; Hong et al., 2020; Munoz et al., 2020). Além dessas, arritmias cardíacas, aperto no peito, dor abdominal, cefaleia e mialgia, também estiveram presentes entre os sinais e sintomas (quadro 2) (Chen et al., 2020; Hong et al., 2020; Zhang, 2020).

Quadro 3 - Sinais e sintomas apresentados por pacientes com COVID-19 e sepse, e população predominante com essas características clínicas. Crato, Ceará, Brasil, 2020.

\begin{tabular}{|l|}
\hline \multicolumn{1}{|c|}{ Sinais e sintomas } \\
\hline População - Neonatos \\
\hline Alimentação reduzida \\
\hline Aumento da frequência cardíaca \\
\hline Congestão nasal \\
\hline Diminuição da saturação de oxigênio \\
\hline Dispneia \\
\hline Hipertermia \\
\hline Hipotensão \\
\hline Hipotermia \\
\hline Letargia \\
\hline Retração subcostal \\
\hline Taquipneia \\
\hline
\end{tabular}




\begin{tabular}{|l|}
\hline População - Adultos \\
\hline Alterações na produção e eliminação de urina \\
\hline Aperto no peito \\
\hline Aumento da frequência cardíaca \\
\hline Cefaleia \\
\hline Diarreia \\
\hline Diminuição da saturação de oxigênio \\
\hline Dispneia \\
\hline Dor abdominal \\
\hline Escarro em excesso \\
\hline Fadiga \\
\hline Febre \\
\hline Hipotensão \\
\hline Letargia \\
\hline Mialgia \\
\hline Náusea \\
\hline Taquipneia \\
\hline Tosse \\
\hline Vômitos \\
\hline \\
População - Idosos \\
\hline Aperto no peito \\
\hline Aumento da frequência cardíaca \\
\hline Bradicardia \\
\hline Cefaleia \\
\hline Diarreia \\
\hline Diminuição da saturação de oxigênio \\
\hline Dispneia \\
\hline Escarro em excesso \\
\hline Fadiga \\
\hline Febre \\
\hline Hipotermia \\
\hline Letargia \\
\hline Náusea \\
\hline Taquipneia \\
\hline Tosse \\
\hline Vômitos \\
\hline
\end{tabular}

Fonte: Autores.

Após análise dos estudos e agrupamentos dos dados sobre os sinais e sintomas mais frequentes apresentados por pacientes com COVID-19 e sepse e/ou choque séptico no momento da admissão e nos dias subsequentes, no ambiente hospitalar, com o auxílio da taxonomia da NANDA-I versão 2018-2020 foram elencados os principais diagnósticos de enfermagem que podem ser aplicados a esse público, de acordo com as características clínicas da população dos estudos selecionados e suas necessidades humanas básicas afetadas.

Foram extraídos todos os sinais e sintomas apresentados nos estudos de pessoas com COVID-19 que apresentaram quadro de sepse e/ou choque séptico, tanto os verbalizados pelos pacientes, como as manifestações verificadas durante a avaliação da equipe de saúde.

Neste contexto, por se tratar de uma doença recente, ainda não foram encontradas publicações que elenquem os diagnósticos de enfermagem para pacientes com o novo coronavírus. Tendo em vista que o curso da doença nos pacientes ainda é incerto e que a sepse é uma das complicações encontradas, podendo levar a desfecho fatal, a elaboração dos DEs pode contribuir para construção de um plano de cuidado sistemático e individualizado para esses pacientes, podendo contribuir para diminuição da mortalidade dos indivíduos acometidos pelo Sars-Cov-2. 
O Quadro 4 traz os principais diagnósticos de enfermagem para pacientes com COVID-19 e sepse, organizados em ordem alfabética, contemplando domínio e classe pertencente, e apresentando fatores relacionados e características definidoras como dispõe na NANDA-I.

Quadro 4 - Diagnósticos de Enfermagem prevalentes nos pacientes com COVID-19 que evoluíram para sepse de acordo com a taxonomia da NANDA-I. Crato, Ceará, Brasil, 2020.

\begin{tabular}{|c|c|c|c|}
\hline Diagnóstico de Enfermagem & Domínio & Classe & $\begin{array}{c}\text { Fatores relacionados/ } \\
\text { Características definidoras }\end{array}$ \\
\hline $\begin{array}{l}\text { Desobstrução ineficaz das vias } \\
\text { aéreas }\end{array}$ & $\begin{array}{l}11 . \\
\text { Segurança/prote } \\
\text { ção }\end{array}$ & 2. Lesão física & $\begin{array}{l}\text { Secreções retidas/ alteração na } \\
\text { frequência respiratória, dispneia e } \\
\text { escarro em excesso }\end{array}$ \\
\hline Dor aguda & 12. Conforto & 1. Conforto físico & $\begin{array}{l}\text { Agente biológico lesivo/ autorrelato } \\
\text { das características da dor e alteração } \\
\text { no parâmetro fisiológico }\end{array}$ \\
\hline $\begin{array}{l}\text { Eliminação } \\
\text { prejudicada }\end{array}$ & $\begin{array}{l}\text { 3. Eliminação e } \\
\text { troca }\end{array}$ & 1. Função urinária & Infecção/ Retenção urinária \\
\hline Fadiga & $\begin{array}{l}4 . \\
\text { Atividade/repou } \\
\text { so }\end{array}$ & $\begin{array}{l}\text { 3. Equilíbrio de } \\
\text { energia }\end{array}$ & $\begin{array}{l}\text { Aumento dos sintomas físicos e } \\
\text { letargia }\end{array}$ \\
\hline Hipertermia & $\begin{array}{l}11 . \\
\text { Segurança/prote } \\
\text { ção }\end{array}$ & $\begin{array}{l}6 . \\
\text { Termorregulação }\end{array}$ & Letargia, taquicardia e taquipneia \\
\hline Hipotermia & $\begin{array}{l}11 . \\
\text { Segurança/prote } \\
\text { ção }\end{array}$ & $\begin{array}{l}6 . \\
\text { Termorregulação }\end{array}$ & Bradicardia \\
\hline Medo & $\begin{array}{l}9 . \\
\text { Enfrentamento/t } \\
\text { olerância ao } \\
\text { estresse }\end{array}$ & $\begin{array}{l}\text { 2. Respostas de } \\
\text { enfrentamento }\end{array}$ & $\begin{array}{l}\text { Reação aprendida a uma ameaça/ } \\
\text { sensação de medo, náusea e vômito }\end{array}$ \\
\hline $\begin{array}{l}\text { Motilidade gastrointestinal } \\
\text { disfuncional }\end{array}$ & $\begin{array}{l}\text { 3. Eliminação e } \\
\text { troca }\end{array}$ & $\begin{array}{l}2 . \quad \text { Função } \\
\text { gastrointestinal }\end{array}$ & $\begin{array}{l}\text { Diarreia, dor abdominal, náusea e } \\
\text { vômito }\end{array}$ \\
\hline Náusea & 12. Conforto & 1. Conforto físico & Ânsia de vômito \\
\hline $\begin{array}{l}\text { Nutrição desequilibrada: } \\
\text { menor do que as necessidades } \\
\text { corporais }\end{array}$ & 2. Nutrição & 1. Ingestão & $\begin{array}{l}\text { Ingestão alimentar insuficiente/ } \\
\text { Ingestão de alimentos menor que a } \\
\text { ingestão diária recomendada (IDR) e } \\
\text { interesse insuficiente pelos } \\
\text { alimentos }\end{array}$ \\
\hline Padrão respiratório ineficaz & $\begin{array}{l}4 . \\
\text { Atividade/repou } \\
\text { so }\end{array}$ & $\begin{array}{l}\text { 4. Respostas } \\
\text { cardiovasculares/ } \\
\text { pulmonares }\end{array}$ & $\begin{array}{l}\text { Fadiga da musculatura respiratória e } \\
\text { hiperventilação/ taquipneia }\end{array}$ \\
\hline Proteção ineficaz & $\begin{array}{l}\text { 1. Promoção da } \\
\text { saúde }\end{array}$ & $\begin{array}{l}\text { 2. Controle da } \\
\text { saúde }\end{array}$ & Dispneia, fadiga e tosse \\
\hline $\begin{array}{l}\text { Risco de desequilíbrio } \\
\text { eletrolítico }\end{array}$ & 2. Nutrição & 5. Hidratação & Diarreia e vômito \\
\hline Termorregulação ineficaz & $\begin{array}{l}11 . \\
\text { Segurança/prote } \\
\text { ção }\end{array}$ & $\begin{array}{l}6 . \\
\text { Termorregulação }\end{array}$ & $\begin{array}{l}\text { Aumento da demanda de oxigênio/ } \\
\text { aumento da frequência respiratória e } \\
\text { aumento da temperatura corporal } \\
\text { acima dos parâmetros normais }\end{array}$ \\
\hline $\begin{array}{l}\text { Ventilação } \quad \text { espontânea } \\
\text { prejudicada }\end{array}$ & $\begin{array}{l}\text { 4. Atividade/ } \\
\text { repouso }\end{array}$ & $\begin{array}{l}\text { 4. Respostas } \\
\text { cardiovasculares/ }\end{array}$ & $\begin{array}{l}\text { Fadiga da musculatura respiratória/ } \\
\text { aumento da frequência cardíaca, }\end{array}$ \\
\hline
\end{tabular}




\begin{tabular}{|l|l|l|l|}
\hline & pulmonares & $\begin{array}{l}\text { diminuição da saturação de oxigênio } \\
(\mathrm{SaO} 2) \text { e dispneia }\end{array}$ \\
\hline Volume de líquidos deficiente & 2. Nutrição & 5. Hidratação & $\begin{array}{l}\text { Aumento da frequência cardíaca e } \\
\text { diminuição da pressão arterial }\end{array}$ \\
\hline
\end{tabular}

Fonte: Autores.

Foram elencados 16 DE da NANDA-I de acordo com o quadro clínico apresentado pelos pacientes, destes, seis mostraram-se mais prevalentes entre os estudos.

Os DEs preeminentes entres os pacientes com COVID-19, sepse e/ou choque séptico foram: Ventilação espontânea prejudicada (domínio 4 - atividade/ repouso, classe 4 - respostas cardiovasculares/pulmonares) relacionada a fadiga da musculatura respiratória evidenciada por aumento da frequência cardíaca, diminuição da saturação de oxigênio e dispneia; padrão respiratório ineficaz (domínio 4 - atividade/repouso, classe 4 - respostas cardiovasculares/pulmonares) relacionado a fadiga da musculatura respiratória e hiperventilação, evidenciado por taquipneia; risco de desequilíbrio eletrolítico (domínio 2 - nutrição, classe 5 - hidratação) evidenciado por diarreia e vômito.

Os diagnósticos termorregulação ineficaz (domínio 11 - segurança/proteção, classe 6 - termorregulação) relacionada ao aumento da demanda de oxigênio evidenciada por aumento da frequência respiratória e aumento da temperatura corporal acima dos parâmetros normais; volume de líquidos deficiente (domínio 2 - nutrição, classe 5 - hidratação) evidenciado por aumento da frequência cardíaca e diminuição da pressão arterial; e dor aguda (domínio 12 - conforto, classe 1 - conforto físico) relacionada a agente biológico lesivo evidenciada por autorrelato das características da dor e alteração no parâmetro fisiológico, também são os mais aplicados entre os casos relatados.

\section{Discussão}

As manifestações mais comuns avaliadas nos pacientes com COVID-19 no momento da admissão hospitalar foram febre, fadiga, dispneia e tosse, seguidas de aperto no peito, mialgia, dor abdominal, diarreia, náusea e vômito. Além de sintomas respiratórios e gastrointestinais, alterações no padrão alimentar em neonatos e comprometimento renal em pacientes adultos com diminuição na eliminação de urina foram observados (Chen et al., 2020; Hansen et al., 2020; Hong et al., 2020; Munoz et al., 2020).

Segundo informações de outras pesquisas que avaliaram sintomas mais frequentes em pacientes hospitalizados com a COVID-19, febre, tosse, coriza, dor de garganta, dispneia, diarreia e náusea estiveram entre as manifestações mais presentes (Associação de Medicina Intensiva Brasileira, 2020; Ministério da Saúde, 2020).

Não foram encontrados estudos de casos de COVID-19 e sepse em crianças acima de 28 dias de idade, apenas em neonatos, podendo-se inferir que o vírus se expressa de forma branda no público infantil.

Os pacientes com COVID-19 que evoluíram com quadro de sepse ou choque séptico foram principalmente neonatos, adultos e idosos. As características clínicas mais comuns a todos os grupos foram: aumento da frequência cardíaca, diminuição da saturação de oxigênio, dispneia, hipertermia, letargia e taquipneia. Um levantamento sobre a faixa etária dos pacientes internados em enfermarias e na UTI com complicações pela COVID-19 em um hospital de Uberlândia, constatou que nas enfermarias a maior proporção de casos eram de indivíduos de 41 a 69 anos e os pacientes com complicações que necessitaram de cuidados intensivos o maior grupo foi o de idosos com idade de 60 a 69 anos (Figueira, 2020).

De acordo com as manifestações informadas nos estudos, foram elaborados os diagnósticos de enfermagem: ventilação espontânea prejudicada (domínio 4 - atividade/repouso, classe 4 - respostas cardiovasculares/pulmonares) relacionada a fadiga da musculatura respiratória evidenciada por aumento da frequência cardíaca, diminuição da saturação de 
oxigênio (SaO2) e dispneia; e hipertermia (domínio 11 - segurança/proteção, classe 6 - termorregulação) evidenciada por letargia, taquicardia e taquipneia.

Outros diagnósticos de enfermagem respiratórios que se adequaram à situação clínica dos pacientes com SARS-CoV2 e sepse foram: Padrão respiratório ineficaz (domínio 4 - atividade/repouso, classe 4 - respostas cardiovasculares/pulmonares) relacionada a fadiga da musculatura respiratória e hiperventilação, evidenciado por taquipneia; e desobstrução ineficaz das vias aéreas (domínio 11 - segurança/proteção, classe 2 - lesão física) relacionada a secreções retidas evidenciada por alteração na frequência respiratória, dispneia e escarro em excesso.

Estudos realizados com crianças e adultos com diagnóstico de infecções respiratórias agudas e asma revelaram alta prevalência para os diagnósticos de enfermagem: Troca de gases prejudicadas, padrão respiratório ineficaz, desobstrução ineficaz das vias aéreas e ventilação espontânea prejudicada (Aquino et al., 2011; Silveira, Lima \& Lopes, 2008). Por se tratar do comprometimento de uma estrutura que está relacionada diretamente com a oxigenação tissular, diagnósticos de enfermagem respiratórios são prioritários porque trata-se de uma função vital para as pessoas.

Para verificação da gravidade de pacientes internados, um dos sistemas de avaliação utilizado pela equipe de saúde é o escore qSOFA, esse, foi empregado em alguns pacientes com o novo coronavírus no momento da admissão para observar se o escore funciona como uma estratégia para classificação precoce dos casos da doença que apresentaram sepse (Ferreira et al., 2020; Hansen et al., 2020).

O qSOFA surgiu como um escore clínico à beira leito para categorizar clinicamente quadro de sepse em pacientes internados, utilizando alguns parâmetros como frequência respiratória $\geq 22$ irpm, alterações neurológicas (Escala de coma de Glasgow < 15) e pressão arterial sistólica $\leq 100 \mathrm{mmHg}$. Uma pontuação qSOFA $\geq 2$ pode indicar mau prognóstico para pacientes com infecção (Instituto Latino Americano de Sepse, 2018).

Escores são usados para prever um curso grave em pacientes com infecção. Nos casos de pacientes com COVID-19, o sistema de pontuação não pareceu muito adequado para esse fim, pois maioria dos pacientes apresentam sinais e sintomas separados e pontuam parâmetros diferentes no escore que não chegam a atingir o ideal para classificação de sepse, sendo o seu uso pouco provável para pacientes infectados com SARS-CoV-2 (Ferreira et al., 2020; Hansen et al., 2020).

Aproximadamente $5 \%$ dos pacientes infectados precisam de cuidados intensivos, isso se relaciona a alguns fatores que contribuem para o agravamento e mudança no comportamento do vírus no organismo, como: idade acima de 60 anos, comorbidades como diabetes e doenças cardíacas, e crianças em idade perinatal. Essas populações apresentaram maior probabilidade de desenvolver síndrome do desconforto respiratório agudo relativa a diversas causas, dentre elas, resposta do sistema imunológico (Campos, 2020).

Estudos anteriores demonstram que a presença de comorbidades está associada a um curso grave da doença e a maior número de mortes. Pacientes com câncer, por exemplo, além da própria doença e o tratamento provocarem um grau de debilidade nos indivíduos e os expor a infecções em razão de alterações na resposta imunológica, o vírus SARS-CoV-2 potencializa o comprometimento do organismo e contribui para um prognóstico ruim (Thuler \& Melo, 2020). Assim, pacientes com histórico de patologias prévias, bem como, extremos de idade, devem adotar medidas mais restritivas para minimizar a exposição potencial ao SARS-CoV-2.

Outra característica clínica importante encontrada durante a evolução da doença nos pacientes com COVID-19 foi a hipotensão, os casos que desenvolveram choque séptico tiveram que fazer reposição volêmica e administração de drogas vasoativas para tratar a acentuada diminuição da pressão arterial (Munoz et al., 2020; Zhang, 2020; Zhou et al., 2020). Para os pacientes com hipotensão, elencou-se o DE volume de líquidos deficiente (domínio 2 - nutrição, classe 5 - hidratação) evidenciado pelo aumento da frequência cardíaca e diminuição da pressão arterial. 
Os sintomas de pacientes com choque séptico são caracterizados por estado de hipoperfusão que provoca hipóxia, lesões e/ou disfunção orgânica, essas alterações resultam da ação de um agente infeccioso que libera mediadores inflamatórios e toxinas no organismo (Jorge et al., 2016). Isso explica a presença de choque séptico em pessoas infectadas pelo SARS-CoV2.

A reposição volêmica tem por objetivo aumentar a perfusão tecidual, esse procedimento deve ser realizado imediatamente após observação do quadro e avaliação do paciente, a compensação de líquidos precisa ser em grande quantidade e deve ser repetida até que haja concentração volêmica satisfatória, com aumento da pressão arterial e do débito urinário. As drogas vasoativas geralmente são reservadas para pacientes que mesmo com a introdução de líquidos não demonstram recuperação da perfusão adequada para tecidos e órgãos (Instituto Latino Americano de Sepse, 2018).

As pessoas com infecção confirmada para SARS-CoV-2 que desenvolveram sepse relataram além dos sintomas respiratórios, fadiga, letargia, cefaleia, aperto no peito, dor abdominal e mialgia (Chen et al., 2020; Hansen et al., 2020; Hong et al., 2020). Para essas manifestações foram aplicados os diagnósticos de enfermagem de dor aguda (domínio 12 - conforto, classe 1 - conforto físico) relacionada a agente biológico lesivo evidenciada por autorrelato das características da dor e alteração no parâmetro fisiológico, e fadiga (domínio 4 - atividade/repouso, classe 3 - equilíbrio de energia) evidenciada pelo aumento dos sintomas físicos e letargia.

Por se tratar de uma doença nova que gerou grandes mudanças na vida das pessoas e por ser o assunto mais debatido nos últimos meses, sentimentos de incertezas e dúvidas passaram a fazer parte dos dias da população mundial em geral. O diagnóstico de enfermagem medo (domínio 9 - enfrentamento/tolerância ao estresse, classe 2 - respostas de enfrentamento) relacionado a reação aprendida a uma ameaça evidenciado por sensação de medo, náusea e vômito, foi elaborado para os indivíduos que relataram sentimento de ameaça e que se sentiram desafiados ao enfrentar a doença.

Além disso, apesar dos pacientes chegarem ao serviço com queixas frequentes de sintomas gripais, observou-se que outros sistemas do corpo humano podem sofrer com a ação do vírus SARS-CoV-2 dentre eles, o sistema gastrointestinal (Chen et al., 2020). Para os distúrbios náusea, vômito e diarreia, foi proposto o DE: motilidade gastrointestinal disfuncional (domínio 3 - eliminação e troca, classe 2 - função gastrointestinal) evidenciada por presença de diarreia, dor abdominal, náusea e vômito.

Assim, a equipe de enfermagem que está atuando na linha de frente no combate ao controle da infecção pelo SARSCoV-2 e na assistência direta ao paciente acometido por esse vírus, precisam ter um olhar sensível e empregar o raciocínio clínico a cada sintoma apresentado pelos pacientes e relatados no momento da busca por ajuda nos serviços de saúde.

Levando em consideração que tanto a COVID-19 como a sepse são doenças que causam sérios danos ao organismo, conhecer o comportamento de ambas constitui um desafio para os profissionais que atuam no front, considerando que o SarsCoV-2 pode ser importante agente para o desenvolvimento da sepse. Nessa perspectiva, a elaboração de diagnósticos de enfermagem viabilizar um olhar centrado no problema do paciente, mais sensível as necessidades básicas afetadas.

\section{Conclusão}

As principais manifestações clínicas encontradas em pacientes com COVID-19 e sepse foram febre, tosse, dispneia, diminuição da saturação de oxigênio, hipotensão, cefaleia, mialgia, vômito e diarreia. Para esses sinais e sintomas 16 diagnósticos de enfermagem da NANDA I foram elaborados, dos quais seis foram prevalentes.

$\mathrm{Na}$ população dos estudos, os DE dominantes são os intitulados de ventilação espontânea prejudicada, padrão respiratório ineficaz, risco de desequilíbrio eletrolítico, termorregulação ineficaz, volume de líquidos deficiente e dor aguda. Maioria estiveram relacionados as alterações do sistema respiratório, estruturas que são mais afetadas pela ação do vírus.

A identificação desses DE oferece subsídios para elaboração de um cuidar direcionado a atender as necessidades dos pacientes. Considerando que a apresentação e evolução da doença são dinâmicas, é necessário que o planejamento da 
assistência e avaliação das ações sejam realizadas constantemente. O Processo de Enfermagem contribui para esse acompanhamento, a partir de suas etapas guia a equipe de enfermagem para um melhor desempenho na assistência e um manejo adequado das necessidades afetadas dos pacientes com COVID-19 e sepse.

Esta revisão permite uma compreensão de como o vírus SARS-CoV-2 age no organismo humano e as possíveis complicações decorrente de sua infecção, dentre elas, a sepse. Além disso, leva a refletir sobre as diversas formas de manifestação da doença, importância do julgamento clínico profissional durante o atendimento desse público, bem como conhecer a população que demonstrou ser mais susceptíveis para prognósticos ruins, que necessitam de atendimento e intervenções precoces.

As literaturas nacional e internacional apresentam relevantes produções sobre os problemas de saúde ocasionados pela COVID-19 e sepse. No entanto, destaca-se que é preciso o desenvolvimento de novas pesquisas envolvendo as implicações sistêmicas a curto e longo prazo provocadas pela infecção por SARS-CoV-2, bem como estudos que abordem os diagnósticos de enfermagem para esse público, tendo em vista a identificação da ausência de publicações sobre DE para pessoas com COVID-19.

\section{Referências}

Aghdam, M. K., Jafari, N., \& Eftekhari, K. (2020). Novel coronavirus in a 15-day-old neonate with clinical signs of sepsis, a case report. Infectious Diseases, 52(6), 427-429.

Alvim M. (2020). Coronavírus: estudo revela fatores de risco e sintomas em pacientes mortos em hospital na China. https://www.bbc.com/portuguese/geral51810488 .

Aquino, R. D., Fonseca, S. M., Lourenço, E. P. L., Leite, A. L., \& Bettencourt, A. R. C. (2011). Mapeamento dos diagnósticos de enfermagem em uma unidade de pneumologia*. Acta Paul Enferm., 24(11), 192-198.

Associação de Medicina Intensiva Brasileira. (2020). CORONAVÍRUS: Esclarecimentos da AMIB pelo Comitê de Sepse e Infecção. https://www.amib.org.br/fileadmin/user_upload/amib/2020/marco/21/11_CORONAVIRUS_Esclarecimentos_da_AMIB_pelo_Comite_de_Sepse_e_Infeccao. pdf.

Associação Mineira de Epidemiologia e Controle de Infecções. (2020). Situação epidemiológica da Covid-19 (Boletim Epidemiológico 10). https://ameci.org.br/situacao-epidemiologica-da-covid-19/.

Botelho, L. L. R., Cunha, C. C. A., \& Macedo, M. (2011). O Método Da Revisão Integrativa Nos Estudos Organizacionais. Gestão \& Sociedade, 5(11), 121136.

Campos, D. C. C. (2020). Coronavírus em Terapia Intensiva: orientações de abordagem do COVID-19. https://pebmed.com.br/coronavirus-em-terapiaintensiva-orientacoes-de-abordagem-do-covid-19/.

Chen, T., Wu, D., Chen, H., Yan, W., Yang, D., Chen, G., Ma, K., Xu, D., Yu, H., Wang, H., Wang, T., Guo, W., Chen, J., Ding, C., Zhang, X., Huang, J., Han, M., Li, S., Luo, X., Zhao, J., \& Ning, Q. (2020). Clinical characteristics of 113 deceased patients with coronavirus disease 2019: retrospective study. $B M J, 368,1-12$.

Conselho Federal de Enfermagem. (2020) Artigo: Profissionais de saúde em tempos de Covid-19. http://www.cofen.gov.br/artigo-profissionais-de-saude-emtempos-de-covid-19_78151.html.

Dewitte, A., Lepreux, S., Villeneuve, J., Rigothier, C., Combe, C., Ouattara, A., \& Ripoche, J. (2017). Blood platelets and sepsis pathophysiology: A new therapeutic prospect in critical ill patients?. An Intensive Care, 7 (1), 115.

Dias, V. M. C. H., Carneiro, M., Vidal, C. F. L., Cunha, C. A., Lobo, S. M. A., Nunes, R. R., et al. (2020) Orientações sobre Diagnóstico, Tratamento e Isolamento de Pacientes com COVID-19. Grupo Força Colaborativa COVID-19 Brasil. https://sao-paulo.estadao.com.br/blogs/blog-da-garoa/wpcontent/uploads/sites/143/2020/04/covid-recomendacao-forca-tarefa_140420200528.pdf.

Ferreira, A. M., Rocha, E. M., Lopes, C. T., Bachion, M. M., Lopes, J. L., Barros, A. L. B. L. (2016). Diagnósticos de enfermagem em terapia intensiva: mapeamento cruzado e Taxonomia da NANDA-I. Rev Bras Enferm., 69(2), 307-15.

Ferreira, M., Blin, T., Collercandy, N., Szychowiak, P., Dequin, P. F., Jouan, Y., \& Guillon, A. (2020). Critically ill SARS-CoV-2-infected patients are not stratified as sepsis by the qSOFA. Ann. Intensive Care, 10(43), 1-3.

Figueira L. (2020) Perfil de pacientes internados com suspeita de Covid-19 em Uberlândia. https://g1.globo.com/mg/triangulomineiro/noticia/2020/03/31/veja-perfil-de-pacientes-internados-com-suspeita-de-covid-19-em-uberlandia.ghtml.

Fineout-Overholt, E., \& Stillwell, S. B. (2011). Asking compelling, clinical questions. In: Melnyk BM, Fineout-Overholt E. Evidence-based practice in nursing \& healthcare: a guide to best practice. Philadelphia: Wolters Kluwer, Lippincot Williams \& Wilkins, 110(3), 25-39. 
Hansen, H. I., Berge, T., Tveita, A., Rønning, E. J., Erno, P. E., Andersen, E. L., Wang, C. H., Myrstad, M. (2020). COVID-19: Symptoms, course of illness and use of clinical scoring systems for the first 42 patients admitted to a Norwegian local hospital. Tidsskr Nor Laegeforen, 140(7), 1-10.

Hong, K. S., Lee, K. H., Chung, J. H., Shin, K. C., Choi, E. Y., Jin, H. J., Jang, J. G., Lee, W., Ahn, E. Y. (2020) Clinical Features and Outcomes of 98 Patients Hospitalized with SARS-CoV-2 Infection in Daegu, South Korea: A Brief Descriptive Study. Yonsei Med J, 61(5), 431 - 437.

Instituto Latino Americano de Sepse. (2018). Atendimento ao paciente adulto com sepse / choque séptico. https://www.ilas.org.br/assets/arquivos/ferramentas/protocolo-de-tratamento.pdf.

Jorge, R. L. N., Lourenço, L. A., Vieira, L. H. A., Santana, M. N., Pedroso, E. R. P. (2016). Choque séptico. Rev Med Minas Gerais, 26(4), 9-12.

Lee, E., Ng, M. Y., Khong, P. L. (2020). COVID-19 pneumonia: what has CT taught us? Lancet Infect Dis., 20 (4), 384-385.

Martins, S. A. G. (2014). A Importância Do Diagnóstico De Enfermagem Para O Acadêmico. 2014. 19f. Monografia (Graduação em Enfermagem) Faculdade de Ciências e Educação Sena Aires, Valparaíso de Goiás. https://www.senaaires.com.br/wp-content/uploads/2017/05/A-IMPORT\%C3\%82NCIADO-DIAGN\%C3\% 93STICO-DE-ENFERMAGEM-PARA-O-ACAD\%C3\%8AMICO.pdf.

Ministério da Saúde (BR). (2020). Boletim Epidemiológico 01 Centro de Operações de Emergências em Saúde Pública | COE-nCoV. Infecção Humana pelo Novo Coronavírus (2019-nCoV). https://portalarquivos2.saude.gov.br/images/pdf/2020/janeiro/28/Boletim-epidemiologico-SVS-28jan20.pdf.

$\begin{array}{lllllllll}\text { Ministério da } & \text { Saúde } & \text { (BR). (2020). Diretrizes } & \text { Para } & \text { Diagnóstico } & \text { E } & \text { Tratamento Da }\end{array}$ https://portalarquivos.saude.gov.br/images/pdf/2020/April/18/Diretrizes-Covid19.pdf.

$\begin{array}{lllllllll}\text { Ministério da } & \text { Saúde } & \text { (BR). (2020). Diretrizes } & \text { Para } & \text { Diagnóstico } & \text { E } & \text { Tratamento Da }\end{array}$ https://portalarquivos.saude.gov.br/images/pdf/2020/April/18/Diretrizes-Covid19.pdf.

Moher, D., Shamseer, L., Clarke, M., Ghersi, D., Liberati, A., Petticrew, M., Shekelle, P., Stewart, L. A. (2015). Preferred reporting items for systematic review and meta-analysis protocols (PRISMA-P) 2015: elaboration and explanation. BJM, 349, 1-25.

Munoz, A. C., Nawaratne, U., McMann, D., Ellsworth, M., Meliones, J., Boukas, K. (2020) Late-Onset Neonatal Sepsis in a Patient with Covid-19. N Engl J Med, 382(19), 1-2.

Organização Pan-Americana de Saúde; Organização Mundial da Saúde. (2020). OMS afirma que COVID-19 é agora caracterizada como pandemia. https://www.paho.org/bra/index.php?option=com_content\&view=article\&id=6120:oms-afirma-que-covid-19-e-agora-caracterizada-comopandemia\&Itemid=812.

Pereira, A. S., Shitsuka, D. M., Parreira, F. J., Shitsuka, R. (2018). Metodologia da pesquisa científica. Ed. UAB/NTE/UFSM https://repositorio.ufsm.br/bitstream/handle/1/15824/Lic_Computacao_Metodologia-Pesquisa-Cientifica.pdf?sequence=1.

Pimentel, T. G. B. (2019). Assistência De Enfermagem Ao Paciente Com Sepse Em Unidades De Terapia Intensiva. Revista Científica Multidisciplinar Núcleo do Conhecimento, 5(5), 05-16.

Silveira, U. A., Lima, L. H. O., Lopes, M. V. O. (2008). Defined characteristics of the nursing diagnoses ineffective airway clearanceand ineffective breathing pattern in asthmatic children. Rev Rene., 9(4), 152-133.

Thuler LCS, Melo AC. Sars-CoV-2/Covid-19 em Pacientes com Câncer. Revista Brasileira de Cancerologia. 2020; 66(2): 1-2.

Zhang, W. (2020). Imaging changes in severe COVID-19 pneumonia in advanced stage. Intensive Care Med., 46(4), 583-585.

Zhou, F., Yu, T., Du, R., Fan, G., Liu, Y., Liu, Z., Xiang, J., Wang, Y., Song, B., Gu, X., Guan, L., Wei, Y., Li, H., Wu, X., Xu, J., Tu, S., Zhang, Y., Chen, H., Cao, B. (2020). Clinical course and risk factors for mortality of adult inpatients with COVID-19 in Wuhan, China: a retrospective cohort study. The Lancet., 395(Supl 10229), 1054-1062.

Zonta, F. N. S., Velasquez, P. G. A., Velasquez, L. G., Demetrio, L. S., Miranda, D., Silva, M. C. B. D. (2018). Características epidemiológicas e clínicas da sepse em um hospital público do Paraná. R. Epidemiol. Control. Infec., 8(3), 224-231. 